\title{
Persée
}

http://www.persee.fr

\section{Les effets de substitution et de richesse de la théorie du portefeuille : une mise au point}

\author{
Jean-Michel Courtault \\ Revue économique, Année 1992, Volume 43, Numéro 6 \\ p. $983-1006$
}

Voir l'article en ligne

\section{Avertissement}

L'éditeur du site «PERSEE » - le Ministère de la jeunesse, de l'éducation nationale et de la recherche, Direction de l'enseignement supérieur, Sous-direction des bibliothèques et de la documentation - détient la propriété intellectuelle et les droits d'exploitation. A ce titre il est titulaire des droits d'auteur et du droit sui generis du producteur de bases de données sur ce site conformément à la loi n`98-536 du 1 er juillet 1998 relative aux bases de données.

Les oeuvres reproduites sur le site «PERSEE » sont protégées par les dispositions générales du Code de la propriété intellectuelle.

Droits et devoirs des utilisateurs

Pour un usage strictement privé, la simple reproduction du contenu de ce site est libre.

Pour un usage scientifique ou pédagogique, à des fins de recherches, d'enseignement ou de communication excluant toute exploitation commerciale, la reproduction et la communication au public du contenu de ce site sont autorisées, sous réserve que celles-ci servent d'illustration, ne soient pas substantielles et ne soient pas expressément limitées (plans ou photographies). La mention Le Ministère de la jeunesse, de l'éducation nationale et de la recherche, Direction de l'enseignement supérieur, Sous-direction des bibliothèques et de la documentation sur chaque reproduction tirée du site est obligatoire ainsi que le nom de la revue et- lorsqu'ils sont indiqués - le nom de l'auteur et la référence du document reproduit.

Toute autre reproduction ou communication au public, intégrale ou substantielle du contenu de ce site, par quelque procédé que ce soit, de l'éditeur original de l'oeuvre, de l'auteur et de ses ayants droit.

La reproduction et l'exploitation des photographies et des plans, y compris à des fins commerciales, doivent être autorisés par l'éditeur du site, Le Ministère de la jeunesse, de l'éducation nationale et de la recherche, Direction de l'enseignement supérieur, Sous-direction des bibliothèques et de la documentation (voir http://www.sup.adc.education.fr/bib/ ). La source et les crédits devront toujours être mentionnés. 


\title{
Les effets de substitution et de richesse de la théorie du portefeuille
}

Une mise au point

Jean-Michel Courtault *

\begin{abstract}
Nous montrons dans cet article que l'approche mise en avant par $P$. Diamond et $M$. Yaari [1972] pour l'étude des propriétés des demandes d'actifs financiers est la seule qui soit compatible avec une définition correcte des effets de richesse. Nous en déduisons une méthode de compensation des effets de richesse, méthode generalisant et démontrant de façon rigoureuse celle préconisée par G. Davis [1989], qui nous permet de mettre au jour la totalité des propriétés des effets de substitution compensés dans l'incertain. Cette méthode nous permet également d'étudier les proprietés des demandes d'actifs par rapport aux caractéristiques de rendement et de risque des actifs.'
\end{abstract}

\section{INTRODUCTION}

Depuis la suggestion de J. Hicks [1935] d'appliquer la théorie des choix à la demande des actifs monétaires et plus généralement à la demande des actifs financiers, les économistes n'ont cessé de s'intéresser à la question. Il est possible de regrouper les diverses contributions en deux grandes catégories suivant le type de modèle utilisé. D'une part, J. Tobin [1958, 1965], J. Hicks [1962], G. Bierwag et M. Groves [1967], S. Royama et K. Hamada [1967], H. Levy [1973], M. Allingham et M. Morishima [1973], A. Courakis [1974], V. Roley [1983] ont, dans le cadre du modèle Moyenne-Variance, tenté d'intégrer l'approche du consommateur aux comportements financiers des agents. D'autre part, S.-C. Kolm [1966], A. Sandmo [1977], S. Fischer [1972], P. Diamond et M. Yaari [1972] et A. Dalal [1983] se sont efforcés de généraliser les résultats obtenus par les auteurs précédemment cités dans le cadre d'un modèle contingent.

L'objet du présent article est d'examiner, dans le cadre d'un modèle à ensemble fini d'états de la nature, les enseignements que l'on peut tirer de l'approche du consommateur pour la théorie du portefeuille. Lorsqu'il existe un nombre

\footnotetext{
* Je remercie P.-A. Chiappori, F. Clerc, M. Mougeot, P. Raimbourg ainsi qu'un rapporteur anonyme pour leurs commentaires.
} 
d'actifs supérieur ou égal au nombre d'états de la nature (et sous condition de rang), on est dans un contexte de marchés complets. Dans ce cas, le modèle considéré est exactement équivalent à un modèle de décision dans le certain. On peut toujours, moyennant un changement de base, se ramener à un système complet de marchés contingents, formellement identique au modèle habituel du consommateur. Dans ce cas, la distinction substitution/revenu est facile à faire, puisque c'est celle de la théorie traditionnelle. Nous montrerons que dans ce cas particulier la méthode de compensation des effets richesse que nous préconisons se confond avec la méthode habituelle (cf. (24') et (25')). En revanche, dans le cas général d'un marché financier incomplet, il est impossible de se ramener au modèle standard du consommateur. Aussi bien, les résultats qui ont été obtenus ont-ils été jugés décevants ${ }^{1}$.

Un tel échec de la littérature est imputable à l'absence de réflexion méthodologique sur la nature des effets de richesse dans l'incertain. Pourquoi les variations des rendements des actifs auraient-elles des effets richesse comme les variations des prix des biens ont des effets revenu? Dans ce dernier cas, on comprend bien qu'une augmentation du prix d'un bien ait à la fois un effet de substitution négatif puisqu'il devient relativement plus cher et un effet revenu puisqu'on ne peut plus acheter autant de biens qu' auparavant. Une augmentation du rendement d'un actif a bien un effet de substitution positif (négatif s'il s'agit d'une vente à découvert) puisque l'actif (la dette) devient relativement plus (moins) attractif(ve) qu'avant, mais quel effet richesse peut-elle bien avoir puisqu'elle ne permet d'acheter ni plus ni moins d'actifs qu'avant? Elle a cependant bien des effets réels puisqu'il s'ensuit une augmentation (diminution) du niveau de l'utilité de l'investisseur. Identifierat-on alors, comme semblent le faire implicitement les auteurs, l'effet richesse à la variation de l'utilité, ce qui permet de le mesurer comme étant égal à la variation de la richesse investie qu'il est nécessaire de donner à l'agent économique pour maintenir son utilité à son niveau initial? Bien que cette façon de voir les choses soit a priori défendable, il faut néanmoins la récuser puisque les effets de substitution qu'elle permet d'isoler ne possèdent en général aucune propriété. C'est pourquoi nous adopterons la définition suivante à la fois pour l'intérêt économique de ses implications et pour sa simplicité qui est en harmonie profonde avec l' approche utilisée dans la théorie du consommateur. L'effet richesse d'une augmentation d'un rendement d'un actif vient de ce que l'investisseur pourra disposer pour ses besoins de consommation ou d'épargne d'une richesse plus importante en fin de période. In s'agit donc non pas d'un effet de richesse présente mais d'un effet de richesse future. Il s'ensuit que pour faire apparaître les effets purs de substitution d'une modification quelconque dans la structure de la distribution du rendement d'un actif on doit compenser les effets richesse par une variation suffisante, pour maintenir

1. Ainsi pour R. Merton [1990] (p. 33) : « Fischer [1972] et Merton [1982] (p. 611614) utilise l'analyse de statique comparative pour montrer que peu [de résultats] peuvent être obtenus sur la structure des fonctions de demandes optimales de portefeuille sauf si l'on impose des restrictions supplémentaires sur la classe des fonctions d'utilité des investisseurs ou la classe des distributions de probabilité des rendements des actifs ». 
l'utilité constante, de la richesse future dans l(es)' état(s) de la nature concerné(s). Nous verrons que cette méthode de compensation permet d'isoler des effets de substitution qui ont des propriétés similaires aux effets de substitution de la théorie du consommateur.

La méthodologie que nous venons d'esquisser dans le paragraphe précédent est celle qui est implicitement adoptée par P. Diamond et M. Yaari [1972]. Cependant, ces auteurs ont présenté leur approche presque comme une curiosité purement technique et ne se sont pas préoccupés d'en donner une motivation économique. Par ailleurs, ils ne suivent pas toujours la même méthodologie et n'en épuisent pas par là même toutes les conséquences. Dans les applications en particulier, ils suivent la méthodologie couramment accepté. Nous souhaitons par le présent travail, d'une part, compléter de façon systématique les résultats obtenus par P. Diamond et M. Yaari [1972] et, d'autre part, montrer que la méthodologie implicitement et partiellement adoptée par ces auteurs est la seule qui soit scientifiquement valable. Nous déterminerons dans un premier temps la méthode générale de compensation des effets richesse de la theorie de la sélection de portefeuille. Puis, nous utiliserons dans un deuxième temps cette méthode de compensation des effets richesse pour étudier les propriétés des effets de substitution de toute une catégorie d'exercices de statique comparative. Nous étudierons en particulier les propriétés des effets de substitution d'une augmentation additive et multiplicative, à risque constant, du rendement anticipé d'un actif quelconque ainsi que celles de l'effet de substitution d'une variation du risque d'un actif à rendement anticipé constant, le risque d'un actif étant mesuré par la dispersion relative de la distribution de ses rendements. Nous verrons également comment il est possible d'exprimer les effets de richesse future en fonction des effets de richesse présente lorsqu'il existe un actif à rendement certain, ce qui nous permettra de relier nos résultats à ceux des auteurs qui, depuis S.-C. Kolm [1966], ont de très nombreuses fois étudié ces exercices de statique comparative. Enfin, en conclusion, nous analyserons les raisons qui ont poussé les auteurs à ne pas percevoir la vraie nature des effets richesse dans l'incertain.

\section{LES EFFETS DE RICHESSE PRÉSENTE ET FUTURE}

On suppose que l'investisseur ait des préférences rationnelles relativement aux distributions possibles de la richesse finale et qui soient représentables par une fonction d'utilité quasi concave du type :

$$
\mathrm{U}(\mathrm{Y}(1), \ldots, \mathrm{Y}(\mathrm{S}) ; \pi(1), \ldots, \pi(\mathrm{S}))=\bar{u}
$$

où $\mathrm{Y}(s)$ est la richesse qui échoit à l'investisseur dans l'état de la nature $s$ dont la probabilité $\pi(s)$ possède les propriétés suivantes :

$$
\forall s=1, \ldots, \mathrm{S} \quad \pi(s)>0, \quad \sum_{s=1}^{\mathrm{S}} \pi(s)=1
$$


Nous supposons également que la fonction d'utilité est croissante par rapport à $Y(s)$ et que, dans le cas où les préférences sont rationnelles au sens de von Neumann-Morgenstern, elle prend la forme suivante :

$$
\mathrm{U}(\mathrm{Y}(1), \ldots, \mathrm{Y}(\mathrm{S}) ; \pi(1), \ldots, \pi(\mathrm{S}))=\sum_{s=1}^{S} \pi(s) u(\mathrm{Y}(s))
$$

Grâce à sa richesse initiale A, l'investisseur peut se composer un portefeuille parmi $n$ actifs (dettes) $i$ en quantité $x_{i}$ positive (négative) de coutt (rendement) unitaire $p_{i}$ positif qu'il pourra (devra) revendre (racheter) en fin de période au prix positif de $p_{i}(s)$ dans l'état $s$. Ainsi, le problème de l'investisseur étant la maximisation de sa fonction objectif (1) sous sa contrainte de richesse initiale, on peut formaliser le problème de l'investisseur, en négligeant toutes les contraintes de nature institutionnelle visant à limiter ses capacités d'endettement ${ }^{1}$, de la façon suivante :

$$
\text { (I) }\left\{\begin{array}{l}
\operatorname{MaxU}(\mathrm{Y}(1), \ldots, \mathrm{Y}(\mathrm{S})) \\
\text { s.c. } \sum_{i=1}^{n} p_{i} x_{i}=A \\
\sum_{i=1}^{n} p_{i}(s) x_{i}+\mathrm{B}(s) \equiv \mathrm{Y}(s) \quad s=1, \ldots, S
\end{array}\right.
$$

où $\mathrm{B}(s)$ est le revenu non financier qui est perçu par l'investisseur dans l'état $s^{2}$.

Il existe deux types d'effets richesse, et donc deux types d'effets substitution ${ }^{3}$, dans la théorie de la sélection de portefeuille. D'une part, un effet richesse présente que l'on peut qualifier de traditionnel : lorsque le prix d(e)' achat (vente) d'un(e) actif (dette) augmente, l'investisseur voit sa situation rélle, mesurée par le niveau d'utilité atteint, se détériorer (s'améliorer) puisqu'il ne peut plus (puisqu'il peut) acquérir un portefeuille aussi important qu'avant. Et, d'autre part, un effet richesse future spécifique à la théorie économique de l'incertain : lorsque le prix de vente (rachat) d'un(e) actif (dette) augmente dans un certain état de la nature $s$, l'investisseur voit sa situation s'améliorer (se détériorer) en terme réel et même en terme nominal puisqu'il pourra disposer ceteris paribus, c'est-à-dire

1. L'influence des contraintes institutionnelles sur le portefeuille optimal a été étudié correctement par S.-C. Kolm [1966], p. 177-188. Nous les ignorons car elles n'ont qu'un rapport formel et non économique avec les effets richesse et ne feraient donc que compliquer inutilement notre analyse.

2. Dans les exercices de statique comparative, le portefeuille optimal sera évalué pour un revenu non financier nul dans tous les états de la nature. Ce revenu non financier est introduit dans le programme de l'investisseur par l'observateur uniquement dans le but de faciliter sa compréhension du comportement de l'agent et non dans un but descriptif.

3. Il y a en fait autant d'effets richesse de nature différente que d'états de la nature (plus un), soit $\mathrm{S}+1$. Nous ignorerons cependant cette évidence pour des raisons de commodité de langage. 
à composition du portefeuille inchangée, pour ses besoins de consommation et d'investissement futurs d'une richesse future plus (moins) importante dans l'état $s$.

De cette distinction il découle qu'il y a deux façons de compenser les effets richesse pour faire apparaître des effets de substitution possédant des propriétes remarquables. On doit, d'une part, compenser les effets de richesse présente par une variation suffisante, c'est-à-dire maintenant l'utilité à un niveau constant, de la richesse présente $A$, et on doit, d'autre part, compenser les effets de richesse future par une variation suffisante de la richesse future $\mathrm{B}(s)$ dans l'état de la nature où l'effet richesse a été induit.

Nous pouvons maintenant passer à l'application de ces principes. Les conditions du premier ordre relatives au programme (I) s'écrivent :

$$
\begin{gathered}
\sum_{s=1}^{\mathrm{S}} \mathrm{U}_{s}^{\prime}(\mathrm{Y}(1), \ldots, \mathrm{Y}(\mathrm{S})) p_{i}(s)-\lambda p_{i}=0 \quad i=1, \ldots, n \\
A-\sum_{i=1}^{n} p_{i} x_{i}=0
\end{gathered}
$$

où $\lambda$ est le multiplicateur de Lagrange associé à la contrainte de richesse initiale.

En supposant la fonction d'utilite (1) strictement quasi concave, on peut montrer que le système formé par les équations (2) et (3) définit de manière unique le portefeuille optimal, ce qui va nous permettre d'étudier sans ambiguïté les modifications des demandes d' actifs entraînées par la variation exogène des paramètres de prix et de richesse. La différenciation des conditions du premier ordre donne le système d'équations suivant :

$$
\begin{gathered}
\sum_{j=1}^{n} \sum_{s=1}^{\mathrm{S}} \sum_{s^{\prime}=1}^{\mathrm{S}} \mathrm{U}_{s s^{\prime \prime}}^{\prime \prime}(.) p_{i}(s) p_{j}\left(s^{\prime}\right) d x_{j}-p_{i} d \lambda=\lambda d p_{i}- \\
-\sum_{s=1}^{\mathrm{s}} \mathrm{U}_{s}^{\prime}(.) d p_{i}(s)-\sum_{s^{\prime}=1}^{\mathrm{S}} \sum_{s=1}^{\mathrm{S}} \mathrm{U}_{s s^{\prime}}^{\prime \prime}(.) p_{i}(s)\left(d \mathrm{~B}\left(s^{\prime}\right)+\sum_{j=1}^{n} x_{j} d p_{j}\left(s^{\prime}\right)\right) \\
-\sum_{j=1}^{n} p_{j} d x_{j}=\sum_{j=1}^{n} x_{j} d p_{j}-d \mathrm{~A}
\end{gathered}
$$

La résolution de ce système permet de déterminer la sensibilité du portefeuille optimal par rapport aux richesses présente et futures

$$
\begin{gathered}
\frac{\partial x_{k}}{\partial \mathrm{A}}=\frac{-\mathrm{D}_{n+1 k}}{\mathrm{D}} \quad k=1, \ldots, n \\
\frac{\partial x_{k}}{\partial \mathrm{B}(e)}=-\sum_{i=1}^{n} \sum_{s=1}^{\mathrm{S}} \mathrm{U}_{s e}^{\prime \prime}(.) p_{i}(s) \frac{\mathrm{D}_{i k}}{\mathrm{D}} \quad \begin{array}{l}
k=1, \ldots, n \\
e=1, \ldots, \mathrm{S}
\end{array}
\end{gathered}
$$


et par rapport aux prix présents et futurs :

$$
\begin{gathered}
\frac{\partial x_{k}}{\partial p_{j}}=\lambda \frac{\mathrm{D}_{j k}}{\mathrm{D}}+x_{j} \frac{\mathrm{D}_{n+1 k}}{\mathrm{D}} \quad j, k=1, \ldots, n \\
\frac{\partial x_{k}}{\partial p_{j}(e)}=-\mathrm{U}_{e}^{\prime}(.) \frac{\mathrm{D}_{j k}}{\mathrm{D}}-x_{j} \sum_{i=1 s=1}^{n} \sum_{s e}^{\mathrm{s}} \mathrm{U}_{s e}^{\prime \prime}(.) p_{i}(s) \frac{\mathrm{D}_{i k}}{\mathrm{D}} \begin{array}{c}
j, k=1, \ldots, n \\
e=1, \ldots, \mathrm{S}
\end{array}
\end{gathered}
$$

où $\mathrm{D}_{i j}$ est le cofacteur associé à l'élément de la $i^{\text {zme }}$ ligne et de la $j^{\text {tme }}$ colonne du déterminant $\mathrm{D}$ de la matrice $\mathrm{H}$ évaluée à l'optimum :

$$
\mathbf{H}=\left[\begin{array}{ll}
\sum_{s=1}^{\mathrm{s}} \sum_{s^{\prime}=1}^{\mathrm{s}} \mathrm{U}_{s s},(.) p_{i}(s) p_{j}\left(s^{\prime}\right) & -p_{1} \\
-p_{1} \ldots \ldots \ldots \ldots \ldots \ldots . . . & -p_{n}
\end{array}\right]
$$

Il est possible de décomposer les effets bruts de substitution (8) et (9) en un effet de substitution net et un effet richesse. Pour ce faire, il est nécessaire de déterminer la variation de l'utilité de l'investisseur induite par la variation exogène des paramètres :

$$
d \mathrm{U}(.)=\sum_{s=1}^{\mathrm{s}} \mathrm{U}_{s}^{\prime}(.)\left(d \mathrm{~B}(s)+\sum_{j=1}^{n}\left(x_{j} d p_{j}(s)+p_{j}(s) d x_{j}\right)\right)
$$

que l'on peut encore réécrire en tenant compte des conditions optimales du premier ordre (2) et (3).

$$
d \mathrm{U}(.)=\sum_{s=1}^{S} \mathrm{U}_{s}^{\prime}(.)\left(d \mathrm{~B}(s)+\sum_{j=1}^{n} x_{j} d p_{j}(s)\right)+\lambda\left(d \mathrm{~A}-\sum_{j=1}^{n} x_{j} d p_{j}\right)
$$

Nous avons vu précédemment que, pour compenser les effets richesse induits par la variation des données, il était nécessaire et suffisant de faire varier la richesse initiale A ou contingente $B(s)$ suivant le type d'effets richesse induits dans une mesure juste suffisante pour maintenir l'utilité constante. On déduit par conséquent de (10) que les variations compensatrices de la richesse sont données par les formules :

$$
\begin{gathered}
d \mathrm{~A}=\sum_{j=1}^{n} x_{j} d p_{j} \\
d \mathrm{~B}(s)=-\sum_{j=1}^{n} x_{j} d p_{j}(s) \quad s=1, \ldots, \mathrm{S}
\end{gathered}
$$

La compensation de l'effet de richesse présente est donc juste suffisante pour permettre à l'investisseur de conserver son portefeuille initial, alors que la compensation des effets de richesse future permet à l'investisseur d'obtenir la même 
configuration de risque et de rendement avec le portefeuille qui était initialement optimal. En effet, la variation de la richesse finale à portefeuille inchangé s'écrit :

$$
d \mathrm{Y}(s)=d \mathrm{~B}(s)+\sum_{j=1}^{n} x_{j} d p_{j}(s) \quad s=1, \ldots, \mathrm{S}
$$

et est par conséquent nulle lorsque les effets richesse sont compensés de façon adéquate, c'est-à-dire lorsque la richesse $\mathrm{B}(s)$ varie suivant la relation (12). Cela permet de justifier l'intuition formulée, dans un contexte voisin du nôtre, par G. Davis [1989] suivant lequel (p. 135) « la compensation est d'un montant qui assure le même résultat ex-post que celui qui aurait été obtenu avant [la modification des paramètres] ». Cette justification n'est pas superflue puisque, toujours d'après cet auteur (p. 135), « il n'y a cependant aucune raison apparente de préférer cette méthode de compensation plutôt que telle autre ». En effet, si la méthode proposée par Davis lui permet de déterminer le signe de l'influence d'une augmentation de la dispersion du prix de vente aléatoire sur la production d'une entreprise contrairement à la méthode de compensation par rapport à la richesse initiale, elle est en revanche plus compliquée que cette dernière. En effet, on doit convenir ex ante d'une règle qui implique un système de compensation ex post alors que la méthode par la richesse initiale définit ex ante une règle de compensation intervenant ex ante. Toutefois le fait que la règle à laquelle nous aboutissons soit stochastique n'est pas un argument décisif contre son utilisation. Nous avons démontré, au contraire, que cette méthode est la seule qui soit économiquement adaptée au problème de la compensation des effets richesse. On ne peut que déplorer que les phénomènes économiques soient plus difficiles à cerner dans l'incertain que dans le certain. Toutefois, la plus grande complexité à laquelle nous sommes confronté est dans la nature des choses : il n'est par conséquent pas en notre pouvoir de la simplifier.

Nous pouvons maintenant, en substituant les équations (11) et (12) dans le système d'équations (4) et (5), déterminer les effets de substitution compensés des effets de richesse présente (CERP) et future (CERF-s) :

$$
\begin{aligned}
\left.\frac{\partial x_{k}}{\partial p_{j}}\right|_{\text {CERP }} & =\lambda \frac{\mathrm{D}_{j k}}{\mathrm{D}} \\
\left.\frac{\partial x_{k}}{\partial p_{j}^{(s)}}\right|_{\text {CERP-s }} & =-\mathrm{U}_{s}^{\prime}(.) \frac{\mathrm{D}_{j k}}{\mathrm{D}}
\end{aligned}
$$

Nous pouvons, grâce aux équations (6)-(9) et (13)-(14), décomposer les effets bruts de substitution de la façon suivante :

$$
\begin{gathered}
\frac{\partial x_{k}}{\partial p_{j}}=\left.\frac{\partial x_{k}}{\partial p_{j}}\right|_{\text {CERP }}-x_{j} \frac{\partial x_{k}}{\partial \mathrm{A}} \\
\frac{\partial x_{k}}{\partial p_{j}(s)}=\left.\frac{\partial x_{k}}{\partial p_{j}(s)}\right|_{\text {CERF-s }}+x_{j} \frac{\partial x_{k}}{\partial \mathrm{B}(s)}
\end{gathered}
$$

On peut interpréter les relations (15) et (16) de manière approximative en disant que l'augmentation d'une unité du prix initial (final) de l'actif $j$ (dans l'état 
de la nature $s$ ) diminue (augmente) la richesse initiale (finale) de $x_{j}$ unités, ce qui a pour conséquence de faire varier la demande de l'actif $k$ de $-x_{j} \cdot \frac{\partial x_{k}}{\partial \mathrm{A}}\left(+x_{j} \cdot \frac{\partial x_{k}}{\partial \overline{\mathrm{B}}(s)}\right)$. Les effets de substitution compensés des effets richesse expliquant le reste de la variation totale de la demande de l'actif $k$.

Les propriétés des effets de substitution compensés découlent de celles de la matrice $\mathrm{H}$. La matrice hessienne d'une fonction d'utilite quasi concave étant symétrique et semi-définie négative, on déduit des équations (13) (16) les relations suivantes ${ }^{1}$ :

$$
\begin{array}{lr}
\frac{\partial x_{k}}{\partial p_{j}}+x_{j} \frac{\partial x_{k}}{\partial \mathrm{A}}=\frac{\partial x_{j}}{\partial p_{k}}+x_{k} \frac{\partial x_{j}}{\partial \mathrm{A}} & j, k=1, \ldots, n \\
\frac{\partial x_{k}}{\partial p_{k}}+x_{k} \frac{\partial x_{k}}{\partial \mathrm{A}} \leq 0 & k=1, \ldots, n \\
\frac{\partial x_{k}}{\partial p_{j}(e)}-x_{j} \frac{\partial x_{k}}{\partial \mathrm{B}(e)}=\frac{\partial x_{j}}{\partial p_{k}(e)}-x_{k} \frac{\partial x_{j}}{\partial \mathrm{B}(e)} & j, k=1, \ldots, n \\
\frac{\partial x_{k}}{\partial p_{k}(e)}-x_{k} \frac{\partial x_{k}}{\partial \mathrm{B}(e)} \geq 0 & e=1, \ldots, \mathrm{S} \\
\end{array}
$$

La symétrie des effets de substitution compensés des effets de richesse présente permet de classer les actifs en substituts et compléments. Nous dirons des actifs $j$ et $k$ qu'ils sont substituts (complémentaires) suivant que l'on ait :

$$
\left.\frac{\partial x_{k}}{\partial p_{j}}\right|_{\text {CERP }}>(<) 0
$$

En effet, suivant (17') la diminution de la demande compensée de l'actif $j$ s'accompagne alors d'une augmentation (diminution) corrélative de la demande compensée de l'actif $k$.

De même, la symétrie des effets de substitution compensés des effets de richesse future nous autorise à classer les actifs en substituts (compléments) suivant que l'on ait :

$$
\left.\frac{\partial x_{k}}{\partial p_{j}(s)}\right|_{\mathrm{CERF}-\mathrm{s}}<(>) 0
$$

L'inversion des inégalités s'explique par le fait que suivant (18') l'augmentation du prix de revente de l'actif $j$ s' accompagne d'une augmentation de la demande compensée de l'actif $j$. Notons également, comme le montrent les équations (13) et (14), que la classification des actifs en substituts et compléments reste la

1. Les relations (17) et (17') ont été démontrées par S. Fischer [1972] dans le cas où l'investisseur se comporte suivant le critère, de l'espérance de l'utilité et les relations (18) et (18') sont dues à P. Diamond et M. Yaari [1972]. 
même que l'on considère les effets de substitution compensés des effets de richesse présente ou future (quel que soit l'état de la nature envisagée).

Il est également possible de montrer que la substitution est dominante parmi les actifs financiers. On a en effet, en vertu du théorème des cofacteurs étrangers, la relation suivante :

$$
\sum_{k=1}^{n}-p_{k} D_{j k}=0 \quad j=1, \ldots, n
$$

d'où l'on déduit :

$$
\begin{array}{rl}
\left.\sum_{k=1}^{n} p_{k} \frac{\partial x_{k}}{\partial p_{j}}\right|_{\text {CERP }}=0 & j=1, \ldots, n \\
\left.\sum_{s=1}^{\mathrm{S}} \sum_{k=1}^{n} p_{k}(s) \frac{\partial x_{k}}{\partial p_{j}(s)}\right|_{\text {CERF-s }}=0 & j=1, \ldots, n
\end{array}
$$

On obtient cette dernière relation en notant que

$$
\begin{aligned}
\left.\sum_{s=1}^{\mathrm{S}} \sum_{k=1}^{n} p_{k}(s) \frac{\partial x_{k}}{\partial p_{j}(s)}\right|_{\mathrm{CERF}-\mathrm{s}} & =-\sum_{k=1}^{n} \sum_{s=1}^{\mathrm{S}} \mathrm{U}_{s}^{\prime}() p_{k}(s) \frac{\mathrm{D}_{j k}}{\mathrm{D}} \\
& =-\sum_{k=1}^{n} \lambda p_{k} \frac{\mathrm{D}_{j k}}{\mathrm{D}}
\end{aligned}
$$

d'après les conditions du premier ordre (2). On tire des relations (22) et (23) en tenant compte des équations (17') et (18') :

$$
\begin{array}{r}
\left.\sum_{\substack{k=1 \\
k \neq j}}^{n} p_{k} \frac{\partial x_{k}}{\partial p_{j}}\right|_{\text {CERP }} \geq 0 \quad j=1, \ldots, n \\
\left.\sum_{S=1}^{s} \sum_{k=1}^{n} p_{k}(s) \frac{\partial x_{k}}{\partial p_{j}(s)}\right|_{\text {CERF-s }} \leq 0 \quad j=1, \ldots, n \\
k \neq j
\end{array}
$$

On déduit de ces dernières relations que dans le cas particulier où deux actifs financiers seulement sont disponibles, 1 'investisseur les considère comme substituts l'un de l'autre (même s'il y a plus de deux états de la nature).

On déduit également des équations (17), (18), (22) et (23) les propriétés d'homogénéité suivantes :

$$
\sum_{j=1}^{n} p_{j} \frac{\partial x_{k}}{\partial p_{j}}+\mathrm{A} \frac{\partial x_{k}}{\partial \mathrm{A}}=0
$$




$$
\sum_{s=1}^{s} \sum_{j=1}^{n} p_{j}(s) \frac{\partial x_{k}}{\partial p_{j}(s)}+\sum_{s=1}^{s} \mathrm{Y}(s)\left(\frac{-\partial x_{k}}{\partial B(s)}\right)=0
$$

c'est-à-dire le portefeuille optimal est insensible à une multiplication identique des prix initiaux et de la richesse présente; la même propriété étant également valable pour une multiplication de tous les rendements dans tous les états de la nature et de la richesse finale dans toutes les éventualités par un même facteur ${ }^{1}$.

La normalité des actifs n'est pas une notion simple comme dans la théorie du consommateur. Il existe en effet deux grandes catégories de normalité : la normalité présente et la normalité future (contingente à l'état $s$ ). L'actif $j$ est normal (inférieur) par rapport à la richesse présente suivant que l'on ait :

$$
\frac{\partial x_{j}}{\partial A} \geq(\leq) 0
$$

De même l'actif $j$ est normal (inférieur) par rapport à la richesse future dans l'éventualité $s$ suivant que l'on ait :

$$
\frac{\partial x_{j}}{\partial \bar{B}(s)} \geq(\leq) 0 \quad s=1, \ldots, S
$$

Un actif peut, par conséquent, être considéré comme normal par rapport à la richesse future dans tel état de la nature et inférieur par rapport à tel autre. Il est toutefois possible d'établir les relations suivantes entre normalité présente et normalité future ${ }^{2}$ :

$$
\begin{array}{lrl}
\sum_{s=1}^{s} p_{j}(s) \frac{\partial x_{k}}{\partial \mathrm{B}(s)}=p_{j} \frac{\partial x_{k}}{\partial \mathrm{A}} & \text { pour } j \neq k=1, \ldots, n \\
\sum_{s=1}^{s} p_{k}(s) \frac{\partial x_{k}}{\partial \mathrm{B}(s)}=p_{k} \frac{\partial x_{k}}{\partial \mathrm{A}}-1 & k=1, \ldots, n
\end{array}
$$

Pour P. Diamond et M. Yaari [1972] l'intuition qui se cache derrière l'équation (24) peut se formuler de la façon suivante (p. 340) :

« Puisque la consommation dépend des possibilités de consommation (c'est-à-dire de la contrainte budgétaire) et pas de la combinaison particulière des revenus [non financier] et des rendements qui donnent naissance à ces possibilités de consommation, nous voyons que la réponse de la consommation dans l'état $s$ à un changement de A est la même que

1. La richesse finale n'étant pas une variable indépendante cette propriété n'est donnée qu'à titre indicatif.

2. Ces relations s'obtiennent, de même que (21) à partir de l'équation de définition: $\dot{H_{H}} \mathbf{H}^{-1}=\mathrm{I}$, où I est la matrice identité d'ordre $\mathrm{n}+\mathrm{l}$. (24) est la généralisation de l'équation (29) de P. Diamond et M. Yaari [1972]. Le modele utilisé par ces auteurs differe du nôtre de deux façons: d'une part, les prix initiaux des actifs sont égaux à l'unité et, d'autre part, le consommateur peut consommer une partie de sa richesse initiale et consomme l'intégralité de sa richesse finale. 
sa réponse à des changements de $\mathrm{B}(1), \ldots, \mathrm{B}(\mathrm{S})$ qui satisfont la relation $\mathrm{dB}(s)=p_{k}(s) \mathrm{dA}$, pour $k$ donné. Il s'ensuit bien ce que nous venons d'écrire puisque l'effet de ces changements de revenus futurs sur la consommation peut être accompli aussi bien en changeant la quantité détenue de l'actif $k$ suite à un changement du revenu présent. Cela implique que les réponses des quantités d'actifs détenus (sauf pour l'actif $k$ ) à ces deux genres de changement doivent aussi être les mêmes de telle sorte que l'on ait le même changement de consommation. "

Dans le cas particulier où existe un système complet d'actifs contingents, ou de façon équivalente lorsqu'il y a autant d'actifs indépendants que d'états de la nature, il est possible d'exprimer chaque effet de richesse future sur la demande d'un actif particulier en fonction de l'effet de richesse présente sur ce même actif

$$
\begin{gathered}
\frac{\partial x_{k}}{\partial \mathrm{B}(j)}=p_{j} \frac{\partial x_{k}}{\partial \mathrm{A}} \quad j \neq k \\
\frac{\partial x_{k}}{\partial \mathrm{B}(k)}=p_{k} \frac{\partial x_{k}}{\partial \mathrm{A}}-1
\end{gathered}
$$

où $p_{i}$ est le prix unitaire d'un actif Arrow-Debreu rapportant un franc dans le ième état de la nature et rien dans les autres.

Toutefois, bien qu'un système complet de marchés financiers soit une abstraction utile, on ne l'observe pas couramment; c'est pourquoi dans les applications concrètes, nous serons amené à utiliser les relations suivantes :

$$
\begin{gathered}
p_{0}(.) \sum_{s=1}^{s} \frac{\partial x_{k}}{\partial \mathrm{B}(s)}=p_{0} \frac{\partial x_{k}}{\partial \mathrm{A}} \quad k=1, \ldots, n \\
p_{0}(.) \sum_{s=1}^{s} \frac{\partial x_{0}}{\partial \mathrm{B}(s)}=p_{0} \frac{\partial x_{0}}{\partial \mathrm{A}}-1
\end{gathered}
$$

où $p_{0}($.$) est le prix final de l'actif à rendement certain.$

Lorsque l'individu maximise l'espérance de son utilité, la somme des effets de richesse future est nulle si l'aversion absolue au risque est constante. En effet :

$$
\begin{aligned}
\sum_{s=1}^{\mathrm{S}} \frac{\partial x_{k}}{\partial \mathrm{B}(s)} & =-\sum_{i=0}^{n} \mathrm{EU}(\tilde{\mathrm{Y}}) \tilde{p}_{i} \frac{\mathrm{D}_{i k}}{\mathrm{D}} \\
& =\frac{-\mathrm{U}^{\prime \prime}(.)}{\mathrm{U}^{\prime}(.)} \sum_{i=0}^{n} \mathrm{EU}^{\prime}(\tilde{\mathrm{Y}}) \tilde{p}_{i} \frac{\mathrm{D}_{i k}}{\mathrm{D}} \\
& =-\lambda \frac{U^{\prime \prime}(.)}{U^{\prime}(.)} \sum_{i=0}^{n} p_{i} \frac{D_{i k}}{D}=0
\end{aligned}
$$


ce qui entraîne également que les effets de richesse présente sont nuls pour les actifs risqués et que tout le poids de l'ajustement repose sur l'actif sans risque, conformément à (24) et à (25).

Du fait de la contrainte de richesse présente, tous les actifs ne peuvent être simultanément inférieurs ou indépendants par rapport à la richesse présente. Cette même contrainte de richesse présente implique la contrainte suivante que doivent respecter les effets de richesse future :

$$
\sum_{k=1}^{n} \sum_{s=1}^{\mathrm{s}} p_{k}(s) \frac{\partial x_{k}}{\partial \mathrm{B}(s)}=0
$$

Notons enfin que la demande d'un actif sera indépendante de la richesse future $\mathrm{B}(e)$ si l'on a :

$$
\mathrm{U}_{s e}^{\prime \prime}(.) / \mathrm{U}_{s}^{\prime}(.)=\text { constante } \quad \forall s=1, \ldots, \mathrm{S}
$$

En effet, les effets de richesse future s'écrivent alors :

$$
\begin{aligned}
\frac{\partial x_{k}}{\partial \overline{\mathrm{B}}(e)} & =\frac{-\mathrm{U}_{s e}^{\prime \prime}(.)}{\mathrm{U}_{s}^{\prime}(.)} \sum_{i=1}^{n} \sum_{s=1}^{\mathrm{s}} \mathrm{U}_{s}^{\prime}(.) p_{i}(s) \frac{\mathrm{D}_{i k}}{\mathrm{D}} \\
& =\frac{-\mathrm{U}_{s e}^{\prime \prime}(.)}{\mathrm{U}_{s}^{\prime}(.)} \sum_{i=1}^{n} \lambda p_{i} \frac{\mathrm{D}_{i k}}{\mathrm{D}}=0
\end{aligned}
$$

où l'on a successivement utilisé les relations (2) et (21). Dans le cas où l'agent économique se comporte suivant le critère de l'espérance de l'utilité, la condition (27) implique la neutralité au risque.

Le cadre (I) dans lequel nous avons choisi d'étudier les propriétés des demandes d'actifs financiers est particulièrement bien adapté pour faire apparaître simplement les principes qui doivent guider toute recherche en matière de sélection optimale des portefeuilles. Toutefois, certaines des propriétés des demandes d'actifs dépendent, si l'on en croit certains auteurs, du modèle particulier qui a été choisi. Par exemple pour Kolm ([1966], p. 174-175) même les effets-prix initiaux ne possèdent aucune propriété remarquable. Cet auteur a choisi de mettre en lumière l'influence des rendements nets des actifs sur les choix de l'investisseur plutôt que celle des rendements bruts, où le rendement net de l'actif $i$ dans l'état $s, z_{i}$ (s), est défini comme la différence entre le rendement brut $p_{i}(\mathrm{~s})$ de l'actif $i$ dans l'état $s$ et son prix d'achat $p_{i}$. Si nous avions choisi un tel cadre, nous aurions été amené à modifier de la façon suivante la procédure de compensation des effets richesse induits par la variation exogène du prix initial des actifs. Il n'est en effet dans ce cas plus suffisant de compenser les effets induits de richesse présente puisqu'une variation du prix initial d'un actif quelconque induit également des 
effets de richesse future ${ }^{\mathfrak{l}}$. Un tel phénomène devient évident lorsqu'on écrit de façon formelle le programme de l'investisseur. Celui-ci devient :

$$
\text { (II) }\left\{\begin{array}{l}
\operatorname{MaxU}(\mathrm{Y}(1), \ldots, \mathrm{Y}(s)) \\
\text { s.c. } \sum_{i=1}^{n} p_{i} x_{i}=A \\
\sum_{i=1}^{n}\left(p_{i}+z_{i}(s)\right) x_{i}+\mathrm{B}(s) \equiv \mathrm{Y}(s) \quad s=1, \ldots, S
\end{array}\right.
$$

La différenciation totale des conditions du premier ordre donne le système d'équations suivant :

$$
\begin{aligned}
\sum_{j=1}^{n} \sum_{s=1}^{\mathrm{S}} \sum_{s^{\prime}=1}^{\mathrm{S}} U_{s s}^{\prime \prime}(.) p_{i}(s) p_{j}\left(s^{\prime}\right) d x_{j}-p_{i} d \lambda= & \\
=\left(\lambda-\sum_{s=1}^{\mathrm{S}} \mathrm{U}_{s}^{\prime}(.)\right) & d p_{i}-\sum_{s=1}^{\mathrm{S}} \sum_{s^{\prime}=1}^{\mathrm{S}} \mathrm{U}_{s s^{\prime \prime}}(.) p_{i}(s)\left(d B\left(s^{\prime}\right)+\right. \\
& \left.+\sum_{j=1}^{n} x_{j}\left(d p_{j}+d z_{j}\left(s^{\prime}\right)\right)\right)-\sum_{s=1}^{\mathrm{S}} \mathrm{U}_{s}^{\prime}(.) d z_{i}(s) \\
& -\sum_{j=1}^{n} p_{j} d x_{j}=\sum_{j=1}^{n} x_{j} d p_{j}-d \mathrm{~A}
\end{aligned}
$$

La résolution de ce système permet de déterminer la sensibilité du portefeuille optimal par rapport aux prix initiaux :

$$
\frac{\partial x_{k}}{\partial p_{j}}=\left(\lambda-\sum_{s=1}^{\mathrm{S}} \mathrm{U}_{s}^{\prime}(.)\right) \frac{\mathrm{D}_{j k}}{\mathrm{D}}+x_{j} \frac{\mathrm{D}_{n+1 k}}{\mathrm{D}}-x_{j} \sum_{i=1}^{n} \sum_{s=1}^{\mathrm{S}} \sum_{s^{\prime}=1}^{\mathrm{S}} \mathrm{U}_{s s^{\prime}}^{\prime \prime}(.) p_{1}(s) \frac{\mathrm{D}_{i k}}{\mathrm{D}}
$$

1. Il est préférable de considérer les choses sous cet angle plutôt que comme Kolm qui, en définissant la richesse finale de la façon suivante: $\mathrm{Y}(s) \equiv \mathrm{A}+\sum_{i=1}^{n} z_{i}(s) x_{i}+$ $+\mathrm{B}(s) \quad s=1, \ldots, \mathrm{S}$ est amené à considérer que la richesse initiale a des effets de richesse présente et future. Pour isoler les effets de substitution compensés, il faudrait alors faire accompagner la variation de la richesse présente par une même variation de la richesse future dans tous les états de la nature égale en valeur absolue et de sens opposé. De cette façon, l'effet de richesse présente induit par la variation de $p_{i}$ est compensé par la variation de la richesse présente, et les effets de richesse future induits par cette dernière sont compensés par la variation des revenus non financier. On peut vérifier aisément que les effets de substitution ainsi isolés sont égaux à (13) et par conséquent possèdent les propriétés (17) et (17'). 
La sensibilité du portefeuille optimal par rapport à la richesse présente et future est donnée respectivement par les équations (6) et (7).

Si l'on compense les effets richesse induits par la variation du prix initial de l'actif $j$ par une variation de la richesse présente et des richesses futures dans la mesure donnée par les équations (11) et (12) respectivement, la résolution du système d'équations (28) et (29) nous donne alors l'effet de substitution compensé des effets richesse présente et future (CERPF) :

$$
\left.\frac{\partial x_{k}}{\partial p_{j}}\right|_{\text {CERPF }}=\left(\lambda-\sum_{s=1}^{\mathrm{s}} \mathrm{U}_{s}^{\prime}(.)\right) \frac{\mathrm{D}_{j k}}{\mathrm{D}}
$$

Nous pouvons ainsi, grâce aux équations (6), (7), (30) et (31), décomposer les effets bruts de substitution de la façon suivante :

$$
\frac{\partial x_{k}}{\partial p_{j}}=\left.\frac{\partial x_{k}}{\partial p_{j}}\right|_{\text {CERPF }}-x_{j}\left(\frac{\partial x_{k}}{\partial \mathrm{A}}-\sum_{s=1}^{\mathrm{s}} \frac{\partial x_{k}}{\partial \mathrm{B}(s)}\right)
$$

La variation totale de la demande de l'actif $k$ suite à une variation unitaire du prix initial de l'actif $j$ est donnée approximativement par la somme de la variation de la demande à richesse réelle inchangée et de la variation de la demande induite par la variation de la richesse réelle de l'investisseur. En effet, lorsque le prix de l'actif $j$ augmente d'une unité, la richesse initiale diminue de $x_{j}$ unités (puisque c'est le cout supplémentaire que doit supporter l'investisseur pour conserver le même portefeuille), ce qui a pour conséquence de faire varier la demande de l'actif $k$ de $-x_{j} \frac{\partial x_{k}}{\partial \mathrm{A}}$ et la richesse finale augmente de $\mathrm{x}_{j}$ unités dans tous les états de la nature, ce qui fait varier la demande de l'actif $k$ de $x_{j} \sum_{s=1}^{s} \frac{\partial x_{k}}{\partial \mathrm{P}(s)}$.

On déduit des relations (31) et (32) la propriété suivante :

$$
\frac{\partial x_{k}}{\partial p_{j}}+x_{j}\left(\frac{\partial x_{k}}{\partial \mathrm{A}}-\sum_{s=1}^{s} \frac{\partial x_{k}}{\partial \mathrm{B}(s)}\right)=\frac{\partial x_{j}}{\partial p_{k}}+x_{k}\left(\frac{\partial x_{j}}{\partial \mathrm{A}}-\sum_{s=1}^{s} \frac{\partial x_{j}}{\partial \mathrm{B}(s)}\right)
$$

c'est-à-dire les effets croisés de prix compensés des effets de richesse présente et future sont symétriques. De plus, dans le cas où l'utilité marginale d'une unité monétaire présente $\lambda$ est supérieure à l'utilité marginale d'une unité monétaire future et également certaine $\left(\sum_{s=1}^{S} U_{s}^{\prime}().\right)$, on a aussi :

$$
\frac{\partial x_{k}}{\partial p_{k}}+x_{k}\left(\frac{\partial x_{k}}{\partial \mathrm{A}}-\sum_{s=1}^{\mathrm{s}} \frac{\partial x_{k}}{\partial \mathrm{B}(s)}\right) \leq 0
$$

Il peut sembler assez paradoxal que les effets de substitution compensés des effets de richesse présente et future du modèle (II) ne soient pas identiques aux effets de substitution compensés des effets de richesse présente du modèle (I). 
En effet, dans les deux cas on a compensé tous les effets richesse induits par une même variation du prix initial de l'actif $j$, on aurait donc pu penser a priori que l'effet sur la demande soit identique. Ce paradoxe s'explique toutefois aisément une fois que l'on a remarqué qu'une même variation du prix initial d'un actif $j$ ne correspond pas au même exercice de statique comparative dans les versions (I) et (II) du modèle de sélection de portefeuille. En effet, dans la seconde version, une variation donnée du prix initial d'un actif quelconque entraîne également une variation identique dans tous les états de la nature du rendement final de cet actif, ce qui n'est pas le cas dans la première version. Puisque les effets bruts de substitution d'une variation du prix initial d'un actif ne correspondent pas à la même chose dans les deux versions du modèle il n'y a aucune raison pour que les effets de substitution compensés des effets richesse soient égaux.

Une fois que l'on a remarqué d'où provient la différence entre les effets bruts de substitution des modèles (I) et (II), il devient facile de relier l'un à l'autre les effets de substitution compensés des effets richesse induits par une variation du prix initial de l'actif $j$. Pour obtenir à partir de (II) les effets purs de substitution du modèle (I), donnés par (13), il suffit de considérer que l'augmentation de $p_{j}$ est accompagnée d'une diminution égale du rendement net de l'actif $j$ dans tous les états de la nature. On a en effet dans ce cas :

$$
\frac{\partial x_{k}}{\partial p_{j}}+x_{j} \frac{\partial x_{k}}{\partial \mathrm{A}}-\sum_{s=1}^{\mathrm{s}} \frac{\partial x_{k}}{\partial z_{j}(s)}=\lambda \frac{\mathrm{D}_{j k}}{\mathrm{D}}
$$

Et, par conséquent, on peut écrire avec S.-C. Kolm ([1966], p. 178) :

$$
\frac{\partial x_{k}}{\partial p_{j}}+x_{j} \frac{\partial x_{k}}{\partial \mathrm{A}}-\sum_{s=1}^{s} \frac{\partial x_{k}}{\partial z_{j}(s)}=\frac{\partial x_{j}}{\partial p_{k}}+x_{k} \frac{\partial x_{j}}{\partial \mathrm{A}}-\sum_{s=1}^{s} \frac{\partial x_{j}}{\partial z_{k}(s)}
$$

c'est-à-dire « les effets croisés de prix compensés de leurs effets de richesse [présente] compensés de leurs effets de rendement sont égaux ».

De même, pour obtenir les effets purs de substitution du modèle (II), donnés par (31), à partir de la formulation du modèle (I), il suffit de postuler que la fonction des anticipations des rendements bruts est de la forme $e^{1}$ :

$$
p_{j}(s)=p_{j}+f(.) \quad \begin{aligned}
& j=1, \ldots, n \\
& s=1, \ldots, \mathrm{S}
\end{aligned}
$$

où dans les arguments de $f\left(\right.$.) ne figurent ni $p_{j}$ ni $p_{j}(s)$. Dans ce cas, l'effet pur de substitution d'une augmentation de $p_{j}$ est bien donné par l'équation (31) ${ }^{2}$.

1. La première version du modèle de portefeuille nous semble préférable à la seconde car elle nous laisse libre de postuler la forme de la fonction d'anticipation des rendements.

2. En effet, dans l'exemple précédent la variation d'un seul paramètre était la cause de la création de $\mathrm{S}+1$ effets richesse que nous avons compensé un à un en nous conformant aux relations (11) et (12). Dans le cas présent, les effets richesse sont crés par la variation simultanée et dépendante de $S+1$ paramètres. La compensation des effets richesse induits obéit de même aux relations (11) et (12), ce qui nous permet d'isoler l'effet pur de substitution et de constater qu'il est identique à (31). 
Dans le cas où l'élasticité des anticipations des rendements par rapport aux prix initiaux est unitaire, c'est-à-dire :

$$
\log p_{j}(s)=\log p_{j}+f(.)
$$

l'effet pur de substitution sur la demande de l'actif $k$ d'une augmentation multiplicative du prix initial de l'actif $j$ est nul. En effet, il est égal à :

$$
\left(\lambda p_{j}-\sum_{s=1}^{\mathrm{s}} \mathrm{U}_{s}^{\prime}(.) p_{j}(s)\right)^{\mathrm{D}_{j k}}
$$

qui s'annule d'après les conditions du premier ordre pour un optimum (2). La variation de la demande étant la résultante des effets richesse uniquement est par conséquent imprévisible en général.

\section{LES EFFETS DE RENDEMENT ET LES EFFETS DE RISQUE}

Nous avons vu dans la section précédente que les seules prédictions que l'on puisse déduire de l'hypothèse de rationalité de l'investisseur se réduisent, dans le cadre du modèle (I) ; aux relations (17)-(18') et (22)-(23). Il est malheureusement impossible de tester ces restrictions puisqu'elles supposent une information qui n'est pas accessible à l'observateur. C'est pourquoi nous allons nous intéresser dans cette section aux propriétés des demandes d'actifs relativement à des variations du risque des actifs et à des variations du rendement espéré des actifs à risque constant. De plus, en supposant l'existence d'un actif sans risque, il nous sera possible de relier les effets de richesse future induits par ces modifications particulières de la distribution des rendements aux effets de richesse présente conformément aux équations (24") et (25"). Ces propriétés se déduisant des propriétés fondamentales des demandes optimales (17)-(18') et (22), (23) permettent de tester celles-ci de manière indirecte. Notons également qu' $a$ priori nous ne pouvons nous attendre à ce que les effets de substitution que nous allons obtenir étant composé à partir des effets de substitution élémentaires (14) possèdent, eux aussi, nécessairement des propriétés de symétrie et de positivité ${ }^{1}$. Par ailleurs, même si les prédictions que nous allons obtenir ne sont pas facilement testables, même si elles ne peuvent en aucune manière constituer un test définitif des propriétés fondamentales dont elles découlent logiquement, il est malgré tout inté-

1. On ne doit pas, par conséquent, comme semblent le suggérer certains auteurs, exiger que l'analogie entre la théorie du consommateur et la théorie du portefeuille soit telle que l'on obtienne également des propriétés de symétrie pour tous les effets croisés de substitution (en particulier relativement au risque). Si on étudiait dans la théorie du consommateur les propriétés des demandes de biens par rapport à des variations simuitanées et dépendantes des prix de plusieurs biens, il est probable que l'on ne pourrait en mettre en évidence aucune. II n'y a, par conséquent, aucune raison d'en attendre davantage de la theorie du portefeuille. 
ressant en soi de connaître les réactions probables des agents économiques face à des variations des paramètres de risque et de rendement des actifs. En particulier, nous serons en mesure de dire si un agent économique augmente (diminue) la quantité d'un actif dont le rendement espéré (risque) s'accroît, lorsqu'on a compensé l'investisseur des effets richesse induits par la modification intervenue dans la structure des rendements de l'actif.

Nous allons envisager après S.-C. Kolm ([1966] p. 175) les modifications structurelles suivantes :

a) le rendement de l'actif $j$ varie de la même quantité dans toutes les éventualités, c'est-à-dire :

$$
\forall s=1, \ldots, S \quad d p_{j}(s)=d p_{j}(.)
$$

b) les rendements de l'actif $j$ dans les diverses éventualités varient tous dans la même proportion, c'est-à-dire :

$$
\forall s=1, \ldots, S \quad d p_{j}(s)=\varepsilon_{j} p_{j}(s)
$$

c) les rendements de $j$ dans les diverses éventualités subissent une dilatation proportionnelle ${ }^{1}$ centrée en leur moyenne $\bar{p}_{j}$ et laissant celle-ci inchangée, c'està-dire :

$$
\forall s=1, \ldots, S \quad d p_{j}(s)=\rho_{j}\left(p_{j}(s)-\bar{p}_{j}\right)
$$

La variation de la demande de l'actif $k$ suite à une variation additive certaine du rendement de l'actif $j$ s'écrit :

$$
\frac{\partial x_{k}}{\partial p_{j}(.)}=-\sum_{s=1}^{\mathrm{S}}\left(\mathrm{U}_{s}^{\prime}(.) \frac{\mathrm{D}_{j k}}{\mathrm{D}}+x_{j} \sum_{i=1}^{n} \sum_{s^{\prime}=1}^{\mathrm{s}} \mathrm{U}_{s s^{\prime}}^{\prime \prime}(.) p_{i}(s) \frac{\mathrm{D}_{i k}}{\mathrm{D}}\right)
$$

ou encore en tenant compte de (7) et (14) :

$$
\frac{\partial x_{k}}{\partial p_{j}(.)}=\sum_{s=1}^{\mathrm{s}}\left(\left.\frac{\partial x_{k}}{\partial p_{j}(s)}\right|_{\mathrm{CERF}-\mathrm{s}}+x_{j} \frac{\partial x_{k}}{\partial \mathrm{B}(s)}\right)
$$

L'effet de substitution compensé d'une augmentation additive certaine du rendement de l'actif $j$ s'écrit par conséquent :

$$
\left.\frac{\partial x_{k}}{\partial p_{j}(.)}\right|_{\mathrm{CERF}}=\left.\sum_{s=1}^{\mathrm{s}} \frac{\partial x_{k}}{\partial p_{j}(s)}\right|_{\mathrm{CERF}-\mathrm{s}}=-\sum_{s=1}^{\mathrm{S}} \mathrm{U}_{s}^{\prime}(.) \frac{\mathrm{D}_{j k}}{\mathrm{D}}
$$

En vertu des propriétés des effets de substitution compensés des effets de richesse future dans l'état $s$, il est facile de montrer que les effets de substitution compensés des effets richesse induits par une variation certaine d'un actif quelconque sont symétriques et défini positifs :

$$
\frac{\partial x_{k}}{\partial p_{j}(.)}-x_{j} \frac{\partial x_{k}}{\partial \mathrm{B}(.)}=\frac{\partial x_{j}}{\partial p_{k}(.)}-x_{k} \frac{\partial x_{j}}{\partial \mathrm{B}(.)} \quad j, k=1, \ldots, n
$$

1. C'est un cas particulier d'un accroissement du risque au sens de Rothschild et Stiglitz; cf. M. Rothschild et J. Stiglitz [1970] et J. Ingersoll [1987], p. 114-124. 


$$
\frac{\partial x_{k}}{\partial p_{k}(.)}-x_{k} \frac{\partial x_{k}}{\partial \mathrm{B}(.)} \geq 0 \quad k=1, \ldots, n
$$

Lorsque le rendement de tous les actifs augmente d'un même montant certain, la demande compensée de l'actif $k$ ne varie pas :

$$
\left.\sum_{j=1}^{n} \frac{\partial x_{k}}{\partial p_{j}(.)}\right|_{\mathrm{CERF}}=0
$$

Lorsqu'il existe un actif à un rendement certain, on peut exprimer l'effet de richesse future en fonction de l'effet de richesse présente. Ce qui nous permet d'écrire ${ }^{1}$ :

$$
\begin{gathered}
\frac{\partial x_{k}}{\partial p_{j}(.)}-x_{j} \frac{p_{0}}{p_{0}(.)} \frac{\partial x_{k}}{\partial \mathrm{A}}=\frac{\partial x_{j}}{\partial p_{k}(.)}-x_{k} \frac{p_{0}}{p_{0}(.)} \frac{\partial x_{j}}{\partial \mathrm{A}} \quad j, k \neq 0 \\
\frac{\partial x_{k}}{\partial p_{0}(.)}-\frac{x_{0}}{p_{0}(.)} p_{0} \frac{\partial x_{k}}{\partial \mathrm{A}}=\frac{\partial x_{0}}{\partial p_{k}(.)}-\frac{x_{k}}{p_{0}(.)}\left(p_{0} \frac{\partial x_{0}}{\partial \mathrm{A}}-1\right)
\end{gathered}
$$

On déduit de ces relations que lorsqu'on compense la variation de l'utilité par une variation de la richesse initiale, on obtient la symétrie des effets croisés de substitution pour les actifs risqués mais pas pour l'actif sans risque. De même, l'effet propre de substitution est positif pour les actifs risqués et de signe indéterminé pour l'actif sans risque. Pour A. Dalal, cette dissymétrie vient de ce que « dans un modèle de portefeuille un actif sans risque et un actif risqué sont qualitativement différents car ce dernier a une dimension supplémentaire - le risque - qui est absent du premier, et par conséquent il n'y a aucune raison de croire que les fonctions de demande pour les actifs risqués et sans risque aient des propriétés semblables » (p. 360). II en découle également qu' « en général aucune relation de substituabilité/complémentarité dénuée d'ambiguüté ne peut être obtenue pour une paire d'actif sans risque et risqué ${ }^{2} »$.

Notons également que dans le cas de l' espérance de l'utilité les effets de richesse future induits par une variation sans risque du rendement des actifs sont nuls lorsque l'aversion absolue au risque est constante.

La variation de la demande, suite à une variation multiplicative du rendement de l'actif $j$, s'écrit :

$$
\frac{\partial x_{k}}{\partial \varepsilon_{j}}=-\sum_{s=1}^{\mathrm{s}}\left(\mathrm{U}_{s}^{\prime}(.) p_{j}(s) \frac{\mathrm{D}_{j k}}{\mathrm{D}}+x_{j} \sum_{i=1}^{n} \sum_{s^{\prime}=1}^{\mathrm{S}} \mathrm{U}_{s s^{\prime}}^{\prime \prime}(.) p_{i}\left(s^{\prime}\right) p_{j}(s) \frac{\mathrm{D}_{i k}}{\mathrm{D}}\right)
$$

1. Cf. (24") et (25").

2. Cf A. Dalal [1983], p. 363. En outre, la définition de la substituabilité de Dalal est plus compliquée qu'ici puisqu'elle fait intervenir en plus du signe de l'effet croisé de substitution d'un accroissement sans risque des rendements espérés le signe de l'effet croisé de substitution d'une augmentation de la variabilité des rendements. Cf. définition 1 et 2 , p. 361 . 
où l'on a posé arbitrairement $\varepsilon_{j}$ égal à zéro. Il est possible de réccrire l'équation précédente de la façon suivante :

$$
\frac{\partial x_{k}}{\partial \varepsilon_{j}}=\sum_{s=1}^{s} p_{j}(s)\left(\left.\frac{\partial x_{k}}{\partial p_{j}(s)}\right|_{\text {CERF-s }}+x_{j} \frac{\partial x_{k}}{\partial \mathrm{B}(s)}\right)
$$

Par conséquent, l'effet de substitution compensé d'une augmentation multiplicative certaine du rendement de l'actif $j$ s'écrit :

$$
\left.\frac{\partial x_{k}}{\partial \varepsilon_{j}}\right|_{\mathrm{CERF}}=\left.\sum_{s=1}^{s} p_{j}(s) \frac{\partial x_{k}}{\partial p_{j}(s)}\right|_{\mathrm{CERF}-\mathrm{s}}=-\sum_{s=1}^{s} \mathrm{U}_{s}^{\prime}(.) p_{j}(s) \frac{\mathrm{D}_{j k}}{\mathrm{D}}
$$

Les effets de substitution compensés des effets richesse induits par une variation multiplicative du rendement du jème actif ne sont pas symétriques ${ }^{1}$. Ils sont toutefois de même signe :

$$
\text { signe }\left(\left.\frac{\partial x_{k}}{\partial \varepsilon_{j}}\right|_{\mathrm{CERF}}\right)=\operatorname{signe}\left(\left.\frac{\partial x_{j}}{\partial \varepsilon_{j}}\right|_{\mathrm{CERF}}\right)
$$

En effet on a :

$$
\left.\frac{\partial x_{k}}{\partial \varepsilon_{j}}\right|_{\text {CERF }}=-\lambda p_{j} \frac{\mathrm{D}_{j k}}{\mathrm{D}}
$$

où l'on a utilisé les conditions du premier ordre pour un maximum (2). De la relation précédente, on déduit également les propriétés suivantes :

$$
\left.\frac{\partial x_{k}}{\partial \varepsilon_{k}}\right|_{\mathrm{CERF}} \geq 0
$$

C'est-à-dire l'effet de substitution sur la demande de l'actif $k$ d'un accroissement multiplicatif du rendement de cet actif est positif.

$$
\left.\sum_{j=1}^{n} \frac{\partial x_{k}}{\partial \varepsilon_{j}}\right|_{\text {CERF }}=0
$$

C'est-à-dire l'effet de substitution sur la demande de l'actif $k$ d'une même augmentation multiplicative du rendement de tous les actifs est nul.

Il est possible d'évaluer l'effet de substitution compensé à partir des effets richesse présente et des effets bruts de substitution. On a, en effet, la relation :

$$
\left.\frac{\partial x_{k}}{\partial \varepsilon_{j}}\right|_{\mathrm{CERF}}=\frac{\partial x_{k}}{\partial \varepsilon_{j}}-p_{j} x_{j} \frac{\partial x_{k}}{\partial \mathrm{A}} \quad \text { pour } j \neq k
$$

1. Notons qu'on obtiendrait des effets de substitution symétriques si l'on avait choisi de faire varier proportionnellement tous les rendements par rapport à un même actif de référence, c'est-à-dire:

$$
\forall s=1, \ldots, S \quad d p_{i}(s)=\varepsilon_{i} p_{j}(s)
$$

ou si l'on avait posé le prix initial de chaque actif égal à l'unité (cf. (40)). 


$$
\left.\frac{\partial x_{k}}{\partial \varepsilon_{k}}\right|_{\text {CERF }}=\frac{\partial x_{k}}{\partial \varepsilon_{k}}-x_{k}\left(p_{k} \frac{\partial x_{k}}{\partial \mathrm{A}}-1\right)
$$

Dans le cas de l'espérance de l'utilité où l'aversion absolue est constante, les effets croisés de richesse induits par une augmentation multiplicative des rendements sont nuls (sauf dans le cas de l'actif sans risque), alors que l'effet propre de richesse sur l'actif certain est nul.

La variation de la demande de l'actif $k$, suite à la dilatation proportionnelle du rendement de l'actif $j$, s'écrit lorsqu'on pose $\rho_{j}$ égal à zéro :

$$
\begin{aligned}
\frac{\partial x_{k}}{\partial \rho_{j}}=-\sum_{s=1}^{\mathrm{S}}\left(\left(\mathrm{U}_{s}^{\prime}(.)\right.\right. & \left.\left(p_{j}(s)-\bar{p}_{j}\right)\right) \frac{\mathrm{D}_{j k}}{\mathrm{D}}+ \\
& \left.+x \sum_{j=1}^{n} \sum_{s^{\prime}=1}^{\mathrm{S}} \mathrm{U}_{s s}^{\prime \prime}(.) p_{i}\left(s^{\prime}\right)\left(p_{j}(s)-\bar{p}_{j}\right) \frac{D_{i k}}{D}\right)
\end{aligned}
$$

Soit encore :

$$
\frac{\partial x_{k}}{\partial \rho_{j}}=\sum_{s=1}^{s}\left(p_{j}(s)-\bar{p}_{j}\right)\left(\left.\frac{\partial x_{k}}{\partial p_{j}(s)}\right|_{\text {CERF-s }}+x_{j} \frac{\partial x_{k}}{\partial \mathrm{B}(s)}\right)
$$

L'effet de substitution compensé d'un accroissement du risque individuel de l'actif $j$ s'écrit :

$$
\begin{aligned}
\left.\frac{\partial x_{k}}{\partial \rho_{j}}\right|_{\text {CERF }}=\sum_{s=1}^{s}\left(p_{j}(s)-\bar{p}_{j}\right) & \left.\frac{\partial x_{k}}{\partial p_{j}(s)}\right|_{\text {CERF-s }} \\
& =-\sum_{s=1}^{s} U_{s}^{\prime}(.)\left(p_{j}(s)-\bar{p}_{j}\right) \frac{D_{j k}}{\mathrm{D}}
\end{aligned}
$$

Là encore, les effets de substitution compensés ne sont pas symétriques ${ }^{1}$. On peut écrire en effet :

$$
\left.\frac{\partial x_{k}}{\partial \rho_{j}}\right|_{\text {CERF }}=-\left(\lambda p_{j}-\bar{p}_{j} \sum_{s=1}^{s} U_{s}^{\prime}(.)\right) \frac{D_{j k}}{\mathrm{D}}
$$

1. On aurait obtenu la symétrie des effets de substitution si l'on avait considéré que les rendements subissent une dilatation proportionnelle par rapport à un même actif de référence, c'est-à-dire :

$$
\forall s=1, \ldots, \mathrm{S} \quad d p_{i}(s)=\rho_{i}\left(p_{j}(s)-\bar{p}_{j}\right)
$$

Dans le cas où il existe un actif sans risque, il y a symétrie si les prix initiaux des actifs sont unitaires et si les rendements des actifs sont égaux en moyenne (cf. A. Dalal, p. 361). 
et lorsqu'il existe un actif à rendement certain :

$$
\left.\frac{\partial x_{k}}{\partial \rho_{j}}\right|_{\mathrm{CERF}}=-\lambda p_{j}\left(1-\frac{\bar{p}_{j} / p_{j}}{p_{0}(.) / p_{0}}\right) \frac{\mathrm{D}_{j k}}{\mathrm{D}}
$$

Pour connaître alors le signe des effets de substitution, il suffit de savoir si les actifs $j$ et $k$ sont substituts ou compléments et de comparer le rendement espéré de l'actif dont la variabilité vient d'augmenter avec celui de l'actif sans risque.

Dans le cas où les préférences de l'investisseur peuvent être représentées par une fonction d'utilité de von Neumann-Morgenstern, les effets de substitution compensés des effets richesse s'écrivent :

$$
\left.\frac{\partial x_{k}}{\partial \rho_{j}}\right|_{\mathrm{CERF}}=-\operatorname{Cov}\left(\mathrm{U}^{\prime}(\tilde{\mathrm{Y}}), \tilde{p}_{j}\right) \frac{\mathrm{D}_{j k}}{\mathrm{D}}
$$

La covariance est négative lorsque l'actif $j$ représente un risque pour l'investisseur ${ }^{1}$. En effet, un actif est risqué lorsque le besoin de richesse, représenté par l'utilité marginale, éprouvé par l'investisseur étant élevé (faible) le rendement de l'actif est faible (élevé). Si l'actif $k$ est risqué, on aura par conséquent :

$$
\left.\frac{\partial x_{k}}{\partial \rho_{k}}\right|_{\text {CERF }} \leq 0
$$

Par ailleurs, si l' actif $k$ est risqué et si les actifs $j$ et $k$ sont substituts (compléments), un accroissement de la variabilité du rendement de l'actif $k$ incite l'investisseur à augmenter (diminuer), mutatis mutandis, la quantité de l'actif $j$. On a en effet :

$$
\left.\frac{\partial x_{j}}{\partial \rho_{k}}\right|_{\mathrm{CERF}} \geq(\leq) 0
$$

Dans le cas où le rendement de l'actif $k$ et l'utilité marginale du portefeuille sont corrélés positivement, l'actif $k$ peut être considéré comme une couverture contre le risque et l'accroissement de sa variabilité a pour conséquence d'augmenter, mutatis mutandis, la demande de l'actif $k$ au détriment des actifs qui lui sont substituts et d'augmenter conjointement la demande des actifs complémentaires.

Les résultats précédents généralisent ceux de J. Hadar et T. Seo ([1990], p. 734-735) lorsque le portefeuille n'est composé que de deux actifs risqués puisque, dans ce cas, ils sont nécessairement substituts : l'augmentation de la variabilité d'un actif doit alors nécessairement augmenter, mutatis mutandis, la demande de l'autre et le budget étant limité cette augmentation doit se faire au détriment du premier actif.

1. Pour une définition voisine du risque individuel d'un actif, cf. R. Merton [1982], p. 614-618. 
On peut évaluer l'effet de substitution compensé lorsqu'il existe un actif à rendement certain. On peut en effet écrire ${ }^{1}$ :

$$
\begin{gathered}
\left.\frac{\partial x_{k}}{\partial \rho_{j}}\right|_{\text {CERF }}=\frac{\partial x_{k}}{\partial \rho_{j}}-p_{j} x_{j}\left(1-\frac{\bar{p}_{j} / p_{j}}{p_{0}(.) / p_{0}}\right) \frac{\partial x_{k}}{\partial \mathrm{A}} \quad j \neq k=1, \ldots, n \\
\left.\frac{\partial x_{k}}{\partial \rho_{k}}\right|_{\text {CERF }}=\frac{\partial x_{k}}{\partial \rho_{k}}-p_{k} x_{k}\left(1-\frac{\bar{p}_{k} / p_{k}}{p_{0}(.) / p_{0}}\right) \frac{\partial x_{k}}{\partial \mathrm{A}}+x_{k} \quad k=1, \ldots, n \\
\left.\frac{\partial x_{0}}{\partial \rho_{k}}\right|_{\mathrm{CERF}}=\frac{\partial x_{0}}{\partial \rho_{k}}-p_{k} x_{k}\left(1-\frac{\bar{p}_{k} / p_{k}}{p_{0}(.) / p_{0}}\right) \frac{\partial x_{0}}{\partial \mathrm{A}}+x_{k} \frac{\bar{p}_{k}}{p_{0}(.)}
\end{gathered}
$$

où l'on a utilisé les relations (24"), (25") et (45).

Notons enfin que dans le cas de l'espérance de l'utilité où l'aversion absolue au risque est constante, les effets croisés de richesse induits par une augmentation du risque individuel des actifs sont nuls pour les actifs risqués.

\section{CONCLUSION}

L'erreur de conception portant sur la nature des effets richesse de la majorité des auteurs vient de la routine qu'a installée la méthode hicksienne de compensation des effets revenu et qui a poussé les économistes à considérer comme équivalentes la compensation des effets de richesse et la compensation de la variation du niveau de l'utilité. Ainsi, A. Dalal [1983], commentant certaines de ses équations, écrit (p. 358) : « les premiers termes [...] sont clairement des effets de substitution puisqu'ils donnent les effets sur les variables de choix d'un changement d'un paramètre lorsque l'utilité espéré est maintenue constante. » Or s'il est bien vrai que la première méthode implique la seconde, la réciproque est fausse : il existe une infinité de façons de maintenir le niveau de l'utilité constant alors qu'une seule permet d'annihiler les effets de richesse. Intuitivement, en effet, la notion d'effet de richesse fait référence à l'idée d'enrichissement ou d'appauvrissement. Toutefois, cet enrichissement ne porte pas nécessairement sur la fortune monétaire d'un individu. Ainsi, dans la théorie du portefeuille, une augmentation du rendement d'un actif dans un état de la nature donné implique un enrichissement sous la forme de richesse contingente à cet état de la nature et non en richesse initiale ou en richesse contingente à un autre état de la nature.

1. L'équation (49) correspond à l'équation (15) de Dalal qui considère également qu'elle est valable pour l'actif non risqué. 


\section{REFERENCES BIBLIOGRAPHIQUES}

Allingham M. G., Morishima M. [1973], « Veblen Effects and Portfolio Selection », dans MORISHIMAM (ed.), Theory of Demand : Real and Monetary, Oxford University Press, Oxford, p. 242-270.

BIERWAG G. O., Groves M. A. [1967], « Slutsky Equations For Assets », Journal of Political Economy, p. 114-127.

CoURAKIs A. S. [1974], « Clearing BankAsset Choice Behaviour : a Mean-Variance Treatment », Oxford Bulletin of Economics and Statistics, p. 173-201.

DalAL A. J. [1983], « Comparative Statics and Asset Substituability/Complementarity in a Portfolio Model : A Dual Approach », Review of Economic Studies, p. 355-367.

DAvis G. K. [1989], « Income and Substitution Effects for Mean-Preserving Spreads », International Economic Review, p. 131-136.

DIAMOND P. A., YAARI M. [1972], «Implications of the Theory of Rationing for Consumer Choice Under Uncertainty », American Economic Review, p. 333-343.

FISCHER S. [1972], «Assets, Contingent Commodities, and the Slutsky Equations », Econometrica, p. 371-385.

HADAR J., SEO T. K. [1990], « The Effects of Shifts in a Return Distribution on Optimal Portfolios », International Economic Review, p. 721-736.

Hicks J. R. [1935], "A Suggestion for Simplifying the Theory of Money », dans HrCKS J. R., Money, Interest and Wages, Basil Blackwell, Oxford, 1982, p. 46-63.

Hrcks J. R. [1962], « Liquidity », Economic Journal, p. 787-802.

INGERSOLL J. E. [1987], Theory of Financial Decision Making, Rowman and Littlefield, Totowa.

KoLM S. C. [1966], Les choix financiers et monétaires, Dunod, Paris.

LEVY H. [1973], «The Demand for Assets Under Conditions of Risk», Joumal of Finance, p. 79-96.

MERTON R. C. [1982], « On the Micrœeconomic Theory of Investment Under Uncertainty », dans ARROW K. J., INTRIIGATOR M. D., Handbook of Mathematical Economics, vol II, North-Holland, Amsterdam, p. 601-669.

MERToN R. C. [1990], « Capital Market Theory and the Pricing of Financial Securities », dans FRIEDMAN B., HAHN F. (eds), Handbook of Monetary Economics, vol I, NorthHolland, Amsterdam, p. 497-581.

Roley V. V. [1983], « Symmetry Restrictions in a System of Financial Asset Demands : Theoretical and Empirical Results », Review of Economics and Statistics, p. 124-130.

ROTHSCHIID M., STIGLITZ J. E. [1970], « Increasing Risk : I. A Definition », Journal of Economic Theory, p. 225-243.

ROYAMA S., HAMADA K. [1967], « Substituability and Complementarity in the Choice of Risky Assets » dans Hester D., TOBIN J. (eds), Risk Aversion in Portfolio Choice, Cowles Foundation Monograph ${ }^{\circ} 19$, New York.

SANDMOA. [1977], «Portfolio Theory, Asset Demand and Taxation : Comparative Statics With Many Assets », Review of Economic Studies, p. 369-379.

ToBIN J. [1958], «Liquidity Preference as Behaviour Toward Risk », Review of Economic Studies, p. 65-86.

ToBan J. [1965], « The Theory of Portfolio Selection » dans HAHN F. H., BrECHLING F. P. R. (eds), The Theory of Interest Rates, MacMillan, Londres, p. 3-51. 
